Anderson, E. S. \& Fraser, A. (1956). J. gen. Microbiol. 15, 225-239

\title{
The Statistical Distribution of Phenotypically Modifiable Particles and Host-range Mutants in Populations of Vi-phage II
}

\author{
By E. S. ANDERSON AND ANTHEA FRASER
}

\author{
Central Enteric Reference Laboratory and Bureau, Public Health Laboratory
}

Service (Medical Research Council), Colindale Avenue, London, N.W. 9

\begin{abstract}
SUMMARY: The distribution of adapted particles of Vi-phage II has been examined. Fluctuation tests in which phage A was grown for a single cycle in type A of $\boldsymbol{S a l}$ monellatyphishowed that phage $\mathbf{E} 1$ particles conformed to a Poisson distribution in a series of small samples and cannot, therefore, be spontaneous mutants of phage $A$. Particles of phage D1, on the other hand, showed a clonal distribution in a similar series of samples and are thus spontaneous host-range mutants of phage A. Phage $\mathbf{E} 1$ reverted to phage A during a single cycle of growth in type A organisms, which confirms that phage $\mathbf{E} 1$ is a phenotypic modification of phage $A$. In contrast, phage D1 was unaltered by a single cycle of propagation in type A organisms and its mutant nature is thus verified. Phage 29 has also been shown to be a host-range mutant of Vi-phage II. The adsorption of phage $A$ to type $E 1$ is lethal to the bacteria, of which only a small proportion support phage growth. The general applicability of these findings to the numerous adaptations of Vi-phage II is discussed.
\end{abstract}

It has been shown recently (Anderson \& Felix, 1952, 1953 $a, b$; Anderson, 1955; Anderson \& Fraser, 1955) that the adaptation of Vi-phage II (Craigie \& Yen, 1938) to the different Vi-types of Salmonella typhi may take any of three forms. First, the adaptation may be a host-induced modification of the type described by Luria \& Human (1952), Bertani \& Weigle (1953), Weigle \& Bertani (1953) and Luria (1953). This change is brought about by the host organisms during phage multiplication, affects every particle formed, and yields phage of which every particle possesses the new host range. When the phage acquires the ability to attack a new host in addition to that on which it was previously propagated, the change can be regarded as adaptive; and the original phage has an approximately constant plating efficiency on the strain on which the new adaptation is to take place. For example, if a phage is already able to attack organism A and is to be adapted to $\mathbf{X}$, its initial efficiency of plating, that is, its titre on $\mathbf{X}$ as a fraction of that on $A$, might be $10^{-4}$. If, after adaptation to $X$, the plating efficiency of the phage on $\mathrm{X}$ becomes 1 , it can be shown that propagation of the phage on $\mathbf{A}$ will reconvert every particle into its original form and its titre on $\mathrm{X}$ compared with that on $\mathrm{A}$ again becomes $10^{-4}$. Such a total change excludes the possibility of the altered host range of the phage being due to the selection of mutants and gave rise to the designation 'host-induced modification' (Luria \& Human, 1952) which we have usually referred to as 'phenotypic modification'. This change may be, as Bertani \& Weigle (1953) point out, adaptive (phage $\mathbf{A} \stackrel{\text { in } \mathbf{X} \text { organisms }}{\longrightarrow}$ phage $\mathbf{A X}$ ) or de-adaptive 
(phage $\mathbf{A X} \stackrel{\text { in } \mathbf{A} \text { organisms }}{\longrightarrow}$ phage $\mathbf{A}$ ). In this example identical symbols are used for the phage and host organisms and it is assumed that, whatever changes of host range the phage may undergo, it will always retain in full the ability to attack organism A. The whole cycle of phenotypic change can be summarized thus:

$$
\text { phage } \mathbf{A} \underset{\text { in } \mathbf{A} \text { organisms }}{\stackrel{\text { in X organisms }}{\rightleftarrows}} \text { phage } \mathbf{A X} \text {. }
$$

It can be seen that this change possesses genetic continuity only when the phage multiplies in the same variety of host organisms. Although it must find a physical representation in the phage deoxyribonucleic acid, the site under host-induced control cannot be regarded as a gene in the accepted sense of the term.

The second type of adaptation which Vi-phage II may undergo expresses itself as a permanent change in host range of the phage. We have referred to this as a 'genotypic' change, and have pointed out in previous publications (Anderson, 1955; Anderson \& Fraser, 1955; Anderson, 1956) that there is evidence that the particles of alternative host range pre-exist in concentrated populations of the wild type of Vi-phage II, which is phage A. Using the same type of symbol as before, phage A may have a plating efficiency of $10^{-4}$ on organism Y. When plaques selected from such a titration are grown with $\mathbf{Y}$ cells, phage $\mathbf{A Y}$ will result, which has an efficiency of plating of 1 on organism $Y$. When this phage is grown in A cells it is propagated unchanged as phage AY; that is, the change from phage $A$ to phage $A Y$ is a mutation affecting the host range of Vi-phage II.

The third type of modification affecting Vi-phage II is a combination of the two forms already described. A host-range mutant may undergo phenotypic modification of the type described earlier.

It has been shown (Anderson, 1955; Anderson \& Fraser, 1955) that the genotypic and phenotypic changes of host range in Vi-phage II are independent of each other and that the phenotypic flexibility of the host-range mutants seems to be as great as that of the wild type of Vi-phage II.

The pattern of susceptibility to Vi-phage II in some Vi-types of Salmonella typhi has been shown to be partly controlled by symbiotic or temperate phages which are known as type-determining phages (Anderson, 1951, Felix \& Anderson, 1951; Anderson \& Felix, 1953b). Structural formulae for the lysogenically determined Vi-types were suggested by Luria (1953) and this suggestion was developed in recent publications (Anderson, 1955; Anderson \& Fraser, 1955). The final proof that a change in the host range of a phage is host-induced or mutational in origin rests on the analysis of single cycles of phage growth in host organisms. Such experiments will be described in the present paper and the statistical distribution of particles showing each type of change will be presented.

\section{METHODS}

Media. 'Difco' broth referred to previously (Anderson \& Felix, 1953a; Anderson \& Fraser, 1955) was used throughout in these experiments; $1.3 \%$ New Zealand powdered agar was incorporated in Difco broth for the prepara- 
tion of routine agar plates and for the basal layer in agar-layer titrations. The surface layer in agar-layer titrations contained $0.45 \%$ of the same agar in Difco broth. One-step growth experiments and phage titrations by the agarlayer technique were carried out by the methods described by Adams (1950).

Fluctuation tests. The phage under examination was mixed with a suspension of the host cells in pre-warmed Difco broth in a volume of $10 \mathrm{ml}$. The suspension consisted of bacteria in the logarithmic phase that had been grown in Difco broth on an agitator. The mixture of phage and bacteria was incubated with agitation at $\mathbf{3 8} .5^{\circ}$ for $15 \mathrm{~min}$. (adsorption tube). Two $\mathrm{ml}$. were then removed and centrifuged at $2125 \mathrm{~g}$. for $10 \mathrm{~min}$. and the resulting supernatant fluid was used for the titration of unadsorbed phage. A sample of the remainder of the adsorption tube was then serially diluted so as to yield final suspensions containing the required number of bacteria. This number varied with the type of experiment and will be indicated at relevant points. The final suspension was distributed in amounts of 0.5 or $1 \mathrm{ml}$. into a number of tubes which were incubated at $\mathbf{3 8} \cdot 5^{\circ}$ for $120 \mathrm{~min}$. Difco agar, inoculated with bacteria of the Vi-type used as the indicator strain in each experiment, was added to each tube to bring the volume to $3 \mathrm{ml}$. and the final percentage of agar to 0.45 . The tubes were then poured on to separate Difco agar plates and incubated overnight.

\section{RESULTS}

An initial one-step growth experiment (Ellis \& Delbrück, 1939; Delbrück, 1940) was carried out to determine the mean burst size of phage $A$ grown on Vi-type A. Fig. 1 shows the results of this experiment. The minimum latent period was $35 \mathrm{~min}$. and the mean burst size about 200 particles. This phage yield is approximately twice that observed by Baron, Formal \& Spilman (1955) with the same phage and Vi-type.

\section{The range of burst size of phage $A$ in type $A$ cells}

The one-step growth experiment gives an estimate of the mean burst size but provides no information concerning the range over which it may be distributed. Experiments were carried out, therefore, to determine this range. Type A cells infected with phage $\mathbf{A}$ in low multiplicity were distributed in a series of 100 tubes so as to give a low probability that any tube contained more than one infected bacterium. The tubes were incubated beyond the latent period and plated on type $A$ to determine the phage yield in each. Table 1 summarizes such an experiment.

$30 \%$ of the tubes in the series summarized in Table 1 yielded counts of between 60 and 300 particles. There was a reasonable probability that these tubes contained single phage-infected bacteria and the range of burst size of phage A grown on type A bacteria evidently lies between these limits. Other fluctuation tests, however, have shown that the lower limit may be as few as 30 particles and that the upper may exceed 400. The burst size varies in different host-range mutants; for example, the mean burst size of phage D1 is about half that of phage $\mathbf{A}$. 


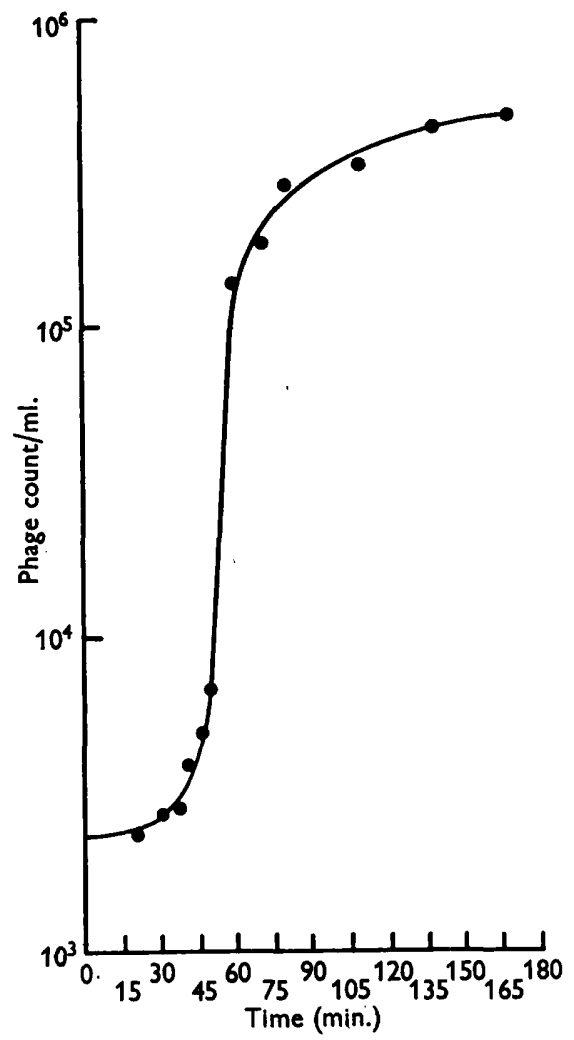

Fig. 1. One-step growth curve of phage A in Vi-type A of Salmonella typhi.

Table 1. Distribution of single bursts of phage A from type A cells

Adsorption tube; $5.0 \times 10^{8}$ phage particles $+3.0 \times 10^{8}$ organisms $/ \mathrm{ml}$.

Phage : organism multiplicity ratio

Free phage after adsorption:

Percentage of phage adsorbed:

$1 \cdot 67: 1$

$1.9 \times 10^{6}$ particles $/ \mathrm{ml}$.

Adsorption tube diluted $0.5 \times 10^{-8}$ and $1.0 \mathrm{ml}$.

99.5

distributed to each of 100 tubes; all tubes incubated at $38 \cdot 5^{\circ}$ for $2 \mathrm{hr}$.

Proportion of tubes not yielding bursts of phage

$m=$ mean number of infected organisms per tube

$$
\begin{aligned}
& =0.54=\mathrm{e}^{-m} \\
& =0.62 \\
& 212
\end{aligned}
$$

Theoretical distribution (Poisson) of number of organisms yielding bursts in 100 tubes:

$\begin{array}{llrl}0 & (54) \text { (observed) } & 3 & 2 \cdot 1 \text { (calculated) } \\ 1 & \mathbf{3 3} \cdot 4 \text { (calculated) } & >3 & 0.5 \text { (calculated) } \\ 2 & 10.0 \text { (calculated) } & & \end{array}$




\section{The distribution of mutants and phenotypically modifiable particles}

If particles of different host range from the main stock occur as mutants in a phage, they will have a clonal distribution in a series of small samples (Luria \& Delbrück, 1943; Luria, 1951). If, on the other hand, they are manifestations of a randomly distributed non-mutational heterogeneity in the phage, or if the phage is qualitatively uniform and the potentiality to adapt any particle to themselves to produce a change in host range is a randomly distributed property of a minority of organisms of the new host, then the plaques of phage

Table 2. The statistical distribution of particles of phage $E 1$ and phage $D 1$ in a population of phage $A$ grown for a single cycle in type $A$ of Salmonella typhi

Efficiency of plating of phage A stock on type D1:

Efficiency of plating of phage A stock on type E1:

$7 \times 10^{8}$ phage A particles $/ \mathrm{ml}$. $\}$ Adsorption tube (AT).

$5 \cdot 2 \times 10^{7}$ type A cells $/ \mathrm{ml}$. Phage: cell multiplicity ratio

A'T incubated at $38.5^{\circ}$ for $15 \mathrm{~min}$. with agitation

Unadsorbed phage in AT:

Percentage of phage adsorbed:

Percentage of cells infected (estimated by count of survivors):

Infective centres on type $A$ before incubation in $10^{-4}$ dilution of $\mathrm{AT}=$ first dilution tube (FDT):

Infective centres on type $\mathbf{A}$ before incubation in dilution containing estimated $10^{3}$ infected cells $/ \mathrm{ml}$. (For distribution in $0.5 \mathrm{ml}$. amounts, incubation and subsequent plating on type D 1):

Infective centres on type $A$ before incubation in dilution containing estimated $2 \cdot 6 \times 10^{2}$ infected cells $/ \mathrm{ml}$. (For distribution in $0.5 \mathrm{ml}$. amounts, incubation and subsequent plating on type $\mathrm{E} 1$ ):

All tubes incubated for $2 \mathrm{hr}$. at $38 \cdot 5^{\circ}$.

Mean burst size:

Efficiency of plating of phage in FDT after incubation:

$$
\begin{array}{ll}
\frac{\text { titre on type D1 }}{\text { titre on type A }} & =1.6 \times 10^{-4} \\
\frac{\text { titre on type E1 }}{\text { titre on type A }} & =2.4 \times 10^{-4}
\end{array}
$$

* Figures corrected for free phage.
$2.1 \times 10^{-4}$

$1 \cdot 1 \times 10^{-3}$

$13 \cdot 5: 1$

$1.25 \times 10^{7} / \mathrm{ml}$.

$98 \cdot 2$

$99 \cdot 8$

$4.4 \times 10^{3} / \mathrm{ml}{ }^{*}$

$7 \cdot 5 \times 10^{2} / \mathrm{ml} . *$

$2.5 \times 10^{2} / \mathrm{ml}$ *

180 particles

with the new host range will show a Poisson distribution in a series of small samples. It was known from previous work (Anderson \& Felix, 1952, 1953 a, $c$; Anderson, 1955; Anderson \& Fraser, 1955) that certain adaptations of Viphage II were purely phenotypic in nature and that these would revert to phage $\mathbf{A}$ (which, in routine test dilution, $\dagger$ lyses only type $\mathbf{A}$ and appears to represent the wild phenotype of Vi-phage II) when grown on type A. Other adaptations of Vi-phage II were known to be genotypically stable. An examination was carried out of the statistical distribution of particles of

† In the Vi-phage typing method each phage is used in the routine test dilution, which is the highest dilution giving confluent or semi-confluent lysis on the homologous type of Salmonella typhi. 
each type of host-range modification in a stock of phage $\mathbf{A}$ grown for a single cycle in type $\mathbf{A}$ organisms. The new host ranges chosen for scrutiny were: E1 (phenotypic) and D1 (host-range mutant). The plating efficiency of phage $A$ on type $E 1$ is about $10^{-3}$; that of phage $A$ on type DI is about $2 \cdot 0 \times 10^{-4}$. The fluctuation tests were carried out as described earlier. Phage $\mathbf{A}$ was adsorbed to type $\mathbf{A}$ bacteria and the suspension was suitably

Table 3. Distribution of plaques after incubation in tubes each containing $0.5 \mathrm{ml}$.

\begin{tabular}{|c|c|}
\hline \multicolumn{2}{|c|}{$\begin{array}{c}\text { From } 3.8 \times 10^{2} \text { infected type A bacteria/ } \\
\text { sample. } \\
\text { Yield of phage }\end{array}$} \\
\hline \multicolumn{2}{|c|}{$\begin{array}{c}\text { Mean total yield/sample plated on } \\
\text { type A: } 1.0 \times 10^{5} \text { particles. }\end{array}$} \\
\hline \multicolumn{2}{|c|}{$\begin{array}{l}\text { Distribution of plaques in } 50 \text { samples } \\
\text { plated on type D1 }\end{array}$} \\
\hline No. of plaques & Frequency \\
\hline $\mathbf{0}$ & 19 \\
\hline $\mathbf{1}$ & 13 \\
\hline 2 & 2 \\
\hline 3 & $\mathbf{0}$ \\
\hline 4 & 2 \\
\hline 5 & 4 \\
\hline 6 & 1 \\
\hline 7 & $\mathbf{0}$ \\
\hline 8 & 1 \\
\hline 14 & 1 \\
\hline 17 & 1 \\
\hline 24 & 1 \\
\hline 33 & 1 \\
\hline 35 & 1 \\
\hline 63 & 1 \\
\hline 95 & 1 \\
\hline 122 & 1 \\
\hline
\end{tabular}

Mean number of plaques/sample on type $\mathrm{DI}=9 \cdot 2$

$\begin{array}{ll}\chi^{2} & =1210 \\ P & =\ll 10^{-8}\end{array}$

Clonal distribution

Mutation rate calculated from the proportion of tubes showing no mutants= $0.97 \times 10^{-5}$.
From 1.3 $\times 10^{2}$ infected type A bacteria/ sample. Yield of phage

Mean total yield/sample plated on type A: $3.5 \times 10^{4}$ particles.

Distribution of plaques in 50 samples plated on type $E 1$

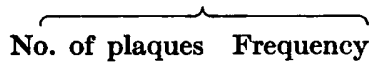

$\begin{array}{rr}0 & 4 \\ 1 & 12 \\ 2 & 13 \\ 3 & 11 \\ 4 & 5 \\ 5 & 4 \\ 6 & 1\end{array}$

Mean number of plaques/sample on type $\mathrm{E} 1=\mathbf{2} \cdot \mathbf{3 4}$ (observed)

$$
\begin{array}{ll}
\chi^{2} & =44.96 \\
P & =0.65
\end{array}
$$

Poisson distribution.

Probability of production of particles plating on type $\mathbf{E} 1$ per reduplication of phage $A=7 \cdot 2 \times 10^{-5}$.

diluted and distributed in $0.5 \mathrm{ml}$. amounts into 120 tubes which were incubated for $2 \mathrm{hr}$. at $38 \cdot 5^{\circ}$. Fifty of the samples were then plated in toto on type $\mathrm{E} 1$, fifty on type $\mathrm{D} 1$ and, after dilution, twenty on type A to estimate the total yield of phage/sample. Tables 2 and 3 show the results of this experiment. It can be seen from Table 3 that the distribution of plaques on type D1 was clonal in character, which suggests that the particles forming plaques on this strain were mutants that arose spontaneously in populations of phage $A$, independently of the presence of type D1 organisms. 
In contrast to these results are those obtained with the samples plated on type E1. Here the plaques showed a Poisson distribution and it may be concluded that they represented particles undergoing phenotypic ('hostinduced') modification after being plated on type E 1 .

Thus, it was possible to demonstrate the presence of host-range mutants and of particles undergoing phenotypic adaptation to a new host in the same stock of phage A resulting from a single cycle of growth in type A organisms.
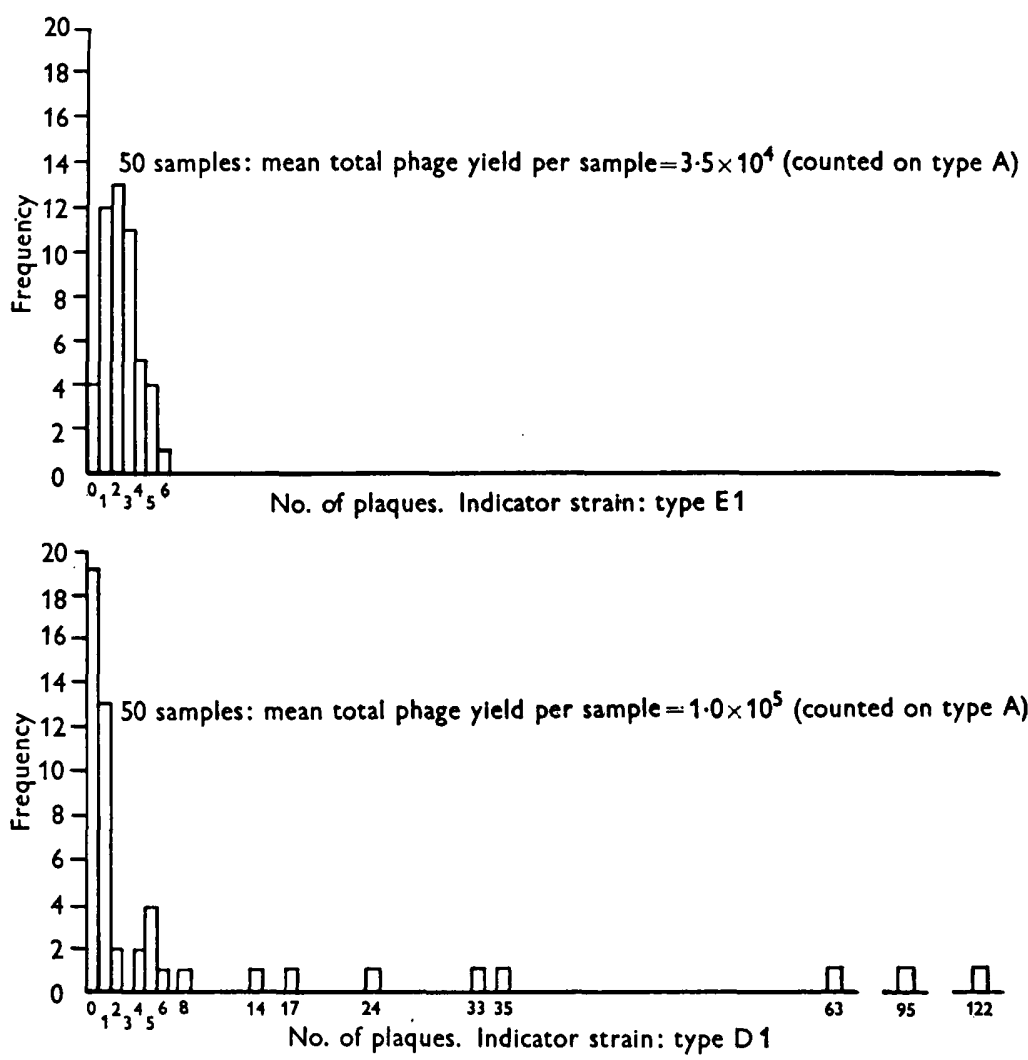

Fig. 2. Fluctuation test (see Tables 2 and 3). Frequency distribution of plaques from single cycle of growth of phage $\mathbf{A}$ in type $\mathbf{A}$ of Salmonella typhi.

Figure 2 is a graphical representation of the distribution of the two types of adapted particle in this experiment. It is known from previously quoted work that both types of change, either separately or in combination, are represented in the Vi-phage typing preparations that have proved so useful in the epidemiology of typhoid fever.

Plate 1 demonstrates effectively the clonal occurrence of the host-range mutant type of particle in phage A stocks. In some of the early experiments the incubation time of the distributed samples was inadequate to ensure that all phage-infected type A bacteria would burst before plating. The period of incubation was later extended to overcome this objection. However, in one of 
the insufficiently incubated batches, an infected organism apparently burst as the surface layer of agar was being poured. The tilting and rotation of the plate distributed the phage particles from the origin of the burst and each produced a plaque on the indicator strain, which was type D1. The total number of plaques in this burst was at least 21 , and it is evident that the group represented a clone of phage DI particles produced by a mutation of phage $\mathbf{A}$ growing in a type $\mathbf{A}$ organism.

From the data in Tables 2 and 3 it is possible to estimate the mutation rate of phage A to phage D1. As the mean total yield of phage/sample in the series plated on type D1 was $1.0 \times 10^{5}$ and the proportion of tubes not showing plaques on type $\mathrm{D} 1$ organisms was $19 / 50=0 \cdot 38$, the mean number of $\mathrm{D} 1$ mutations/tube was $\mathbf{0 . 9 7}$, assuming the frequency of mutation to conform to a Poisson distribution. If, as Luria (1951) suggests, phage multiplies exponentially, the mutation rate to phage $D 1$ per reduplication of phage $A$ in this experiment was $0.97 \times 10^{-5}$. There was a probability of a mean contribution of 1 particle of phage D1/sample from the original stock of phage $A$, but experiments indicated that such particles were unlikely to be able to produce bursts of phage D1 from type A organisms in the presence of the greatly superior numbers of phage $\mathbf{A}$ particles (about 13) infecting the same organisms. The fluctuation test was repeated, using a mean multiplicity of 1 phage $\mathbf{A}$ particle/type A organism; the results were corrected by subtraction of bursts which might have been produced by infection of the type A cells with phage $\mathrm{D} 1$ particles already present in the original stock of phage $A$. The phage multiplicity ratio in this experiment was sufficiently low to allow pre-existing phage D1 particles to multiply in the bacteria they infected. After the correction had been applied, this fluctuation test yielded a figure similar to that given in Table 3 for the mutation rate of phage A to phage D1.

It was found that when phage $\mathbf{A}$ was applied to a lawn of type D1 on agar plates, the host range of a number of single plaques picked at random could be divided into two classes which occurred with approximately equal frequency. Class 1 lysed only type D1, whereas class 2 lysed both types D1 and D5. Table 4 shows the sensitivity of types D1 and D5 to their homologous typing phages and to the two classes of plaques picked from plates on which phage A had been titrated on type D1.

It must be pointed out that phage D 5 is normally prepared by adapting phage A to type D5; it was shown in earlier work (Anderson \& Felix, 1953a) to be a host-range mutant of Vi-phage II. It is clear from Table 4 that class 1 plaques were identical with phage D1, and class 2 with phage D5. Type D1 selects either mutant indifferently because it is as sensitive to phage $\mathrm{D} 5$ as it is to phage D1. This is additional proof that the production of the host-range mutants of Vi-phage II is independent of the presence of the Vi-types of Salmonella typhi which they can lyse. The ability to lyse type D5, which is always accompanied by the ability to lyse type D1, can be regarded as an unselected marker when the indicator strain is type $\mathbf{D} 1$.

It is thus possible that roughly half the mutations of phage $A$ to phage D 1 recorded in Table 3 were to phage D5. The true mutation rate to phage D1 
would thus be about $50 \%$ of the calculated rate. However, no attempt was made to distinguish phage D1 from phage D 5 in the experiment recorded in Tables 2 and 3.

Table 4. Reactions of phages D1 and D5 and of the two classes of plaques picked from adaptations of phage $\boldsymbol{A}$ to type $\boldsymbol{D} 1$ of Salmonella typhi

$\begin{array}{ccc}\begin{array}{c}\text { Indicator } \\ \text { Salmonella } \\ \text { typhi }\end{array} & \overbrace{\text { D1 }}^{\text {Typing phages }} \\ \text { D 1 } & + & \text { D5 } \\ \text { D5 } & - & + \\ +=\text { lysis } ;-=\text { no lysis. } & & +\end{array}$

Plaques picked from plating of phage A on type D1

$\begin{array}{cc}\text { Class } 1 & \text { Class } 2 \\ + & + \\ - & +\end{array}$

\section{Effect on phenotypically changed preparations and host-range mutants of single cycles of growth on type $A$}

In earlier publications (Anderson \& Felix, 1952, 1953 $a, c$; Anderson, 1955; Anderson \& Fraser, 1955; Anderson, 1956) it was shown that the difference between phenotypically-changed particles such as those of phage E1, and genotypically-changed stocks such as phage Dl can be demonstrated by growing each on type $\mathbf{A}$, when the phenotypically-changed stocks revert uniformly to phage $A$ while the host-range mutants, if they have not previously undergone phenotypic modification, are propagated unchanged. These experiments were carried out by titrating the respective phages on type $\mathbf{A}$, and selecting and propagating discrete plaques. The resulting preparations thus represented an unknown number of cycles of phage growth. Single-cycle experiments were carried out in order to demonstrate unequivocally the difference between the two types of adaptation represented by the starting phages. The respective phages were adsorbed to type $\mathbf{A}$ organisms and the suspensions diluted so as to give only a few organisms $/ \mathrm{ml}$. One ml. amounts were distributed into fifty tubes. After incubation for $2 \mathrm{hr}$. to ensure that all infected bacteria had lysed, $\mathbf{0 . 5} \mathrm{ml}$. of each tube was plated on type $\mathbf{A}$, and $0.5 \mathrm{ml}$. on the correct indicator strain for the phage under test: type $\mathbf{E} 1$ for phage $\mathrm{E} 1$ and type $\mathrm{D} 1$ for phage D1. In addition, a dilution containing about 100 infected organisms/ml. was incubated in bulk for $2 \mathrm{hr}$. and titrated on the relevant indicator strains. Tables 5 and 6 show the results of these experiments. Only the findings on the dilutions incubated in bulk are given.

The platings of the multiple small samples, which had a mean content of about five bacteria each, showed that the host range of the phage-yield from individual bacteria agreed exactly with that from the bulk dilution. Thus, it is apparent that the change from phage $\mathbf{E} 1$ to phage $A$, with loss of the ability to multiply in type $\mathrm{E} I$ bacteria, occurred in a single cycle of growth in type A. On the other hand, phage D1 after growth on type A preserved its host range unchanged, retaining an equal efficiency of plating on type $\mathrm{A}$ and D1. 
The experiments summarized in Tables 5 and 6 indicate the justifiability of the authors' earlier assumptions concerning the phenotypic nature of certain of the Vi-phage II adaptations (see Anderson \& Fraser, 1955) and also confirm the stability of host range of those preparations which have been previously

\section{Table 5. Single cycle of growth of phage $E 1$ in type $A$ of Salmonella typhi}

$6.4 \times 10^{8}$ organisms $+4.8 \times 10^{8}$ phage E1 particles $/ \mathrm{ml}$.

After $15 \mathrm{~min}$. adsorption at $\mathbf{3 8} \cdot 5^{\circ}$, the suspension was diluted

$10^{-6}$ and incubated for $2 \mathrm{hr}$. at $38 \cdot 5^{\circ}=$ Second dilution tube (SDT).

Phage adsorbed (\%):

Infective centres in SDT, before incubation, on types

$A$ and E1:

Efficiency of plating of phage E1: $\frac{\text { titre on type E1 }}{\text { titre on type A }}$

Infective centres in SDT after incubation (1) On type A

(2) On type $\mathrm{E}_{1}$

Efficiency of plating of phage $\mathbf{E} 1$ after growth on type $A$

titre on type E 1

titre on type $A$

$\mathbf{9 9} \cdot \mathbf{3}$

$3 \cdot 0 \times 10^{2} / \mathrm{ml}^{*}$

$=\mathbf{1} \cdot \mathbf{0}$

$4.5 \times 10^{4} / \mathrm{ml}^{*}$

$76 / \mathrm{ml}$.

$=1 \cdot 7 \times 10^{-3}$

150 particles

* Figures corrected for free phage.

Table 6. Single cycle of growth of phage $D 1$ in type $A$ of Salmonella typhi

$6.2 \times 10^{8}$ organisms $+6.7 \times 10^{8}$ phage $D 1$ particles $/ \mathrm{ml}$.

After 15 min. adsorption at $38 \cdot 5^{\circ}$, the suspension was diluted

$10^{-6}$ and incubated for $2 \mathrm{hr}$. at $38 \cdot 5^{\circ}=$ Second dilution tube

(SDT)

Phage adsorbed (\%):

Infective centres in SDT, before incubation, on types A

96.0 and D 1:

Efficiency of plating of phage D $1: \frac{\text { titre on type D 1 }}{\text { titre on type A }} \quad=1 \cdot 0$

Infective centres in SDT after incubation

(1) On type A:

(2) On type D1:

Efficiency of plating of phage D 1 after growth on type A

$4 \cdot 3 \times 10^{4} / \mathrm{ml}^{*}$ $4.4 \times 10^{4} / \mathrm{ml}$.

titre on type D 1

titre on type $A$

$=\mathbf{1} \cdot \mathbf{0}$

Mean burst size:

107 particles

* Figures corrected for free phage.

regarded as mutants. It is, of course, known that both changes may be represented in a single preparation of Vi-phage II, and such a phage has a wider host range than those in which purely phenotypic-on-wild-type or purely genotypic changes have occurred, because the host ranges covered by the phenotypic and genotypic changes are summated in each particle. A full discussion of this subject was given by the present authors in a recent paper (Anderson \& Fraser, 1955). 


\section{Effect of adsorption of Vi-phage II preparations on heterologous Vi-types}

Although the adsorption of a phage to an organism to which it can become phenotypically adapted is usually as efficient before adaptation as after, the effect of such adsorption on the host varies with the different phage host systems. For example, Bertani \& Weigle (1953) found that phage P2, isolated from the lysogenic Escherichia coli of Lisbonne and Carrère (Bertani, 1951), when grown on the indicator strain of Shigella shigae (Sh), was unable to lyse E. coli B. Nevertheless, P2Sh, that is, phage P2 adapted to strain Sh, was readily adsorbed by $E$. coli $B$ but did not affect its growth. On the other hand, Luria \& Human (1952) and Luria (1953) found that the phenotypic modification of phage $\mathrm{T} 2$ known as $\mathrm{T}^{*} 2$, which was adsorbed to $E$. coli $\mathrm{B}$ or B/4o (a mutant of $E$. coli B) without causing lysis or phage reproduction, killed the organisms to which it became attached.

Experiments were carried out to determine the effect of the adsorption of phage A on type $\mathbf{E} 1$ of Salmonella typhi using phage:host cell multiplicity ratios of 150:1, 15:1 and 1.5:1. The concentration of type $E 1$ bacteria in the adsorption mixtures was $1.7 \times 10^{8}$. Adsorption was carried out for $15 \mathrm{~min}$. at $38.5^{\circ}$ with agitation. The concentration of unadsorbed phage was then estimated by centrifuging $2 \mathrm{ml}$. of the suspension at $2125 \mathrm{~g}$ for $10 \mathrm{~min}$. and titrating the supernatant on type $A$. The number of surviving bacteria was determined by the method of Miles \& Misra (1938). The total number of infective centres, that is, free phage + infected bacteria yielding phage, was calculated by plate counts on types A and E1. Dilutions were made in chilled broth and all manipulations were completed during the latent period, so that infective centres registering on type $\mathbf{E} 1$ represented bacteria of type $\mathbf{E} 1$ infected in the adsorption mixtures at the commencement of the experiment. The infected organisms which produced plaques on type $\mathbf{E} 1$ would obviously have yielded bursts of phage E1 if they had been allowed to lyse before plating. The actual phage $A$ concentrations used were $2.56 \times 10^{10}, 2.56 \times 10^{9}$ and $2.56 \times 10^{8} / \mathrm{ml}$. The average efficiency of plating of phage A on type E1 is about $10^{-3}$. Theoretically, therefore, the maximum number of particles in the phage $\mathbf{A}$ stocks in the three successive experiments which could function as phage $\mathrm{E} 1$ particles was of the order of $2 \cdot 3 \times 10^{7}, 2 \cdot 3 \times 10^{6}$ and $2 \cdot 3 \times 10^{5} / \mathrm{ml}$. respectively. As the starting concentration of bacteria was $1.7 \times 10^{8}$, not more than $14 \%$ of type $\mathrm{E} 1$ organisms could have been killed by particles of phage $A$ capable of multiplying as phage $E 1$ in the highest phage concentration used. Thus, any appreciable killing effect on type E1 organisms would be due to adsorption of phage $\mathbf{A}$ without subsequent phage multiplication. The results of these experiments are summarized in Table 7. The estimation of the fraction of infected type $\mathrm{E} 1$ bacteria functioning as infective centres was based entirely on the plating results on type $\mathbf{E} 1$ organisms. It is apparent from Table 7 that adsorption of phage $\mathbf{A}$ was lethal to type $\mathrm{E} 1$, although multiplication of the phage occurred in only a minority of organisms. 
Table 7. Lethal effect of phage $A$ on Vi-type $E 1$ of Salmonella typhi

Initial concentration of type $\mathrm{E} 1$ organisms : $1.7 \times 10^{8} / \mathrm{ml}$. Multiplicities of phage Aadsorbed to the bacteria: Expt. 1, 150:1; Expt. 2, 15:1; Expt. 3, 1.5:1. Adsorption: 15min.at 38.5 ${ }^{\circ}$.

tration
Free phage after
adsorption
Phage adsorbed ( $\%)$
Surviving bacteria
Bacteria killed by
phage infection ( $\%$ )
Infective centres on
type E 1
Killed bacteria not
yielding phage $(\%)$

\begin{tabular}{cll} 
Expt. 1 & \multicolumn{1}{c}{ Expt. 2 } & \multicolumn{1}{c}{ Expt. 3 } \\
$2.56 \times 10^{10} / \mathrm{ml}$. & $2.56 \times 10^{9} / \mathrm{ml}$. & $2.56 \times 10^{8} / \mathrm{ml}$. \\
$3.6 \times 10^{8} / \mathrm{ml}$. & $6.2 \times 10^{6} / \mathrm{ml}$. & $4.1 \times 10^{5} / \mathrm{ml}$. \\
& & \\
98.6 & 99.8 & 99.8 \\
$5 \cdot 3 \times 10^{4} / \mathrm{ml}$. & $3.6 \times 10^{7} / \mathrm{ml}$. & $1.3 \times 10^{8} / \mathrm{ml}$. \\
$>99.9$ & $79\left(=1.34 \times 10^{8} / \mathrm{ml}.\right)$ & $24\left(=4.0 \times 10^{7} / \mathrm{ml}.\right)$ \\
& & $3.67 \times 10^{4} / \mathrm{ml}$. \\
$1.25 \times 10^{6} / \mathrm{ml}$. & $5 \cdot 4 \times 10^{4} / \mathrm{ml}$. & \\
$99 \cdot 3$ & $>99.9$ & 99.9
\end{tabular}

\section{DISCUSSION}

When Craigie \& Yen (1938) first described the Vi-phage typing method they suggested that the adapted preparations of Vi-phage II originated from hostrange mutants of the phage that were selected by the specific types of Salmonella typhi on which they could grow. This explanation was rejected by Felix (1949) on the grounds that it was unlikely that two sets of complementary mutants could have been evolved in such widely different organisms as the typhoid bacillus and Vi-phage II. The experiments described in this paper show that the adaptation of Vi-phage II to the various Vi-types of Salmonella typhi consists of the two different processes of host-induced modification and selection of host-range mutants of the phage, which may occur separately or in combination. Phage D1 was chosen at random for scrutiny as a hostrange mutant. An examination of the mutation of phage $A$ to phage 29 has yielded similar results. It is reasonable to conclude, therefore, that all the specific adapted preparations which differ in genotype from phage $\mathbf{A}$ are hostrange mutants of Vi-phage II. Thus, Craigie \& Yen's original hypothesis is partly correct. The demonstration that any preparation of Vi-phage II, either mutant or wild-type, is capable of the same phenotypic plasticity (Anderson \& Fraser, 1955) shows that the phage is also able to extend its host range widely without mutational changes. However, the suggestion put forward by Anderson \& Felix $(1953 a, c)$ that the entire range of Vi-phage II adaptations are modifications of the phage phenotype only is incorrect.

There are at least 21 Vi-types (including two that have recently been discovered) that can be lysed only by host-range mutants of phage A. In 13 of these the Vi-type specificity has been shown to depend partly on lysogenicity (Anderson, 1951, Felix \& Anderson, 1951, Anderson \& Felix, 1953b) and the types concerned can be manufactured artificially by lysogenizing suitable non-lysogenic types* with the correct temperate type-determining phages.

* The term 'non-lysogenic' is used only in relation to the absence of type-determining phages. Temperate phages that do not have a type-determining function are commonly found in Salmonella typhi; these are not considered here. 
Thus, these determining phages evidently offer an obstacle to the multiplication of the wild genotype of Vi-phage II which can only be overcome by hostrange mutants of the Vi-phage. The identity of each type-determining phage decides which mutant of Vi-phage II shall be selected. In some cases the identification of the particular mutant of Vi-phage II selected offers the only known distinguishing feature between determining phages. For example, when type A of Salmonella typhi carries phage $\mathrm{f} 2$ it becomes type 29 (structural formula $A(f 2)$ ). This type is sensitive to, among others, phages 29 and D6. When type $A$ is lysogenized with phage $\mathrm{d} 6$, however, it becomes type $\mathrm{D} 6$, which is structurally $A(d 6)$ and this complex is sensitive to phage $\mathrm{D} 6$ but relatively resistant to phage 29 . Phages 29 and D6 are different host-range mutants of Vi-phage II and which of the two is selected depends on whether the selecting host is carrying phage $\mathrm{f} 2$ or phage $\mathrm{d} 6$. As type 29 is equally sensitive to phages 29 and D6, it is evident that if these mutants occurred at equal rates in phage $A$, they would be selected indiscriminately when phage $A$ was applied to type 29 . However, phage $A$ mutates to phage 29 with considerably higher frequency than it does to phage D6. This gives phage 29 a selective advantage over phage D 6 when concentrated stocks of phage $A$ are applied to type 29. Phages $\mathrm{f} 2$ and $\mathrm{d} 6$ are serologically indistinguishable, show identical properties of lability to heat and chemical agents, have identical host ranges and protect the strains they lysogenize against each other. They are distinguishable only by the identification of the host ranges of the mutants of Vi-phage II selected by the bacteria they have lysogenized.

It has been pointed out (Anderson, 1955; Anderson \& Fraser, 1955) that the lysogenically-determined types can be regarded as consisting of two components: the non-lysogenic precursor of the type; and the type-determining phage that is presumably associated with the bacterial nucleus. The former is responsible for the phenotypic modification of Vi-phage II; the latter blocks the multiplication of the wild genotype of the Vi-phage and determines which host-range mutant of it shall be selected. These two functions are independent of one another and each must be represented physically in both the organism and the Vi-phage. It can thus be postulated that there are two steps in the multiplication of Vi-phage II that are capable of undergoing changes of a definable nature. One, which is responsible for the changes of the phage in phenotype only, is controlled by a complementary site in the host organism that is unaffected by lysogenization with the type-determining phages. The effect of this site on Vi-phage II can be regarded as a permanent characteristic of the host organism. The effect on Vi-phage II of the site in the bacterium controlled by the type-determining phage depends on the particular determining phage concerned. Each determining phage erects a specific barrier to the wild genotype of Vi-phage II that can only be overcome by a particular hostrange mutant or by closely related mutants.

All Vi-types of Salmonella typhi are able to adsorb all adaptations of Viphage II irrespective of their specificity. The experiments with the adsorption of phage $\mathbf{A}$ to type $\mathbf{E} 1$ described in this paper show that such adsorption is only 
followed by phage multiplication when the phage is of suitable phenotype and genotype. This indicates that the steps in Vi-phage II multiplication that are governed by the phenotypic and genotypic characters examined are distinct from, and follow, adsorption of the phage to the host organism.

We are indebted to Dr A. Bernstein and Dr C. C. Spicer for statistical advice and many fruitful discussions, and to Mr F. J. Flynn for valuable technical assistance.

\section{REFERENCES}

Adams, M. H. (1950). Bacterial viruses. In Methods in Medical Research, 2. Chicago: Year Book Publishers.

Anderson, E. S. (1951). The significance of Vi-phage types F1 and F2 of Salmonella typhi. J. Hyg., Camb. 49, 458.

Anderson, E. S. (1955). Consideration of the Vi-phage types of Salmonella typhi on a structural basis. Nature, Lond. 175, 171.

Anderson, E. S. (1956). A new Vi-phage type of Salmonella typhi; with a discussion of methods of preparation of typing phages for new Vi-types. J.gen. Microbiol. $14,676$.

Anderson, E. S. \& Feurx, A. (1952). Variation in Vi-phage II of Salmonella typhi. Nature, Lond. 170, $4,92$.

Anderson, E. S. \& Felix, A. (1953a). 'Degraded Vi strains' and variation in Viphage II of Salmonella typhi. J. gen. Microbiol. 8, 408.

Anderson, E. S. \& Felix, A. (1953b). The Vi type-determining phages carried by Salmonella typhi. J. gen. Microbiol. 9, 65.

Anderson, E. S. \& Felix, A. (1953c). Vi-phage-type specificity and degradation in Salmonella typhi. Proc. V1th Congr. int. Microbiol. Rome, 3, 462.

Anderson, E. S. \& Fraser, A. (1955). The influence of the factors determining Vitype specificity in Salmonella typhi on the adaptation of Vi-phage II. J. gen. Microbiol. 13, 519.

Baron, L. S., Formal, S. B. \& Spilman, W. (1955). Vi-phage-host interaction in Salmonella typhosa. J. Bact. 69, $17 \%$.

Bertani, G. (1951). Studies on lysogenesis. I. The mode of phage liberation by lysogenic Escherichia coli. J. Bact. 62, 293.

Bertani, G. \& Weigle, J. J. (1953). Host controlled variation in bacterial viruseș. J. Bact. 65, 113.

Craigie, J. \& Yen, C. H. (1938). The demonstration of types of B. typhosus by means of preparations of Type II Vi phage. Canad. publ. Hlth J. 29, 448, 484.

Desbrück, M. (1940). The growth of bacteriophage and lysis of the host. J. gen. Physiol. 23, 643.

Ellis, E. L. \& Delbrück, M. (1939). The growth of bacteriophage. J.gen. Physiol. $22,365$.

Felix, A. (1949). The nature of bacteriophages. Proc. IVth Congr. int. Microbiol., Copenhagen, p. 355.

Felix, A. \& Anderson, E. S. (1951). Bacteriophages carried by the Vi-phage types of Salmonella typhi. Nature, Lond. 167, 603.

Luria, S. E. (1951). The frequency distribution of spontaneous bacteriophage mutants as evidence for the exponential rate of phage reproduction. Cold Spr. Harb. Symp. quant. Biol. 16, 463.

Luria, S. E. (1953). Host-induced modifications of viruses. Cold Spr. Harb. Symp. quant. Biol. 18, 237.

LURIA, S. E. \& DeLBrücK, M. (1943). Mutations of bacteria from virus sensitivity to virus resistance. Genetics, $28,491$. 
Journal of General Microbiology, Vol. 15, No. 1

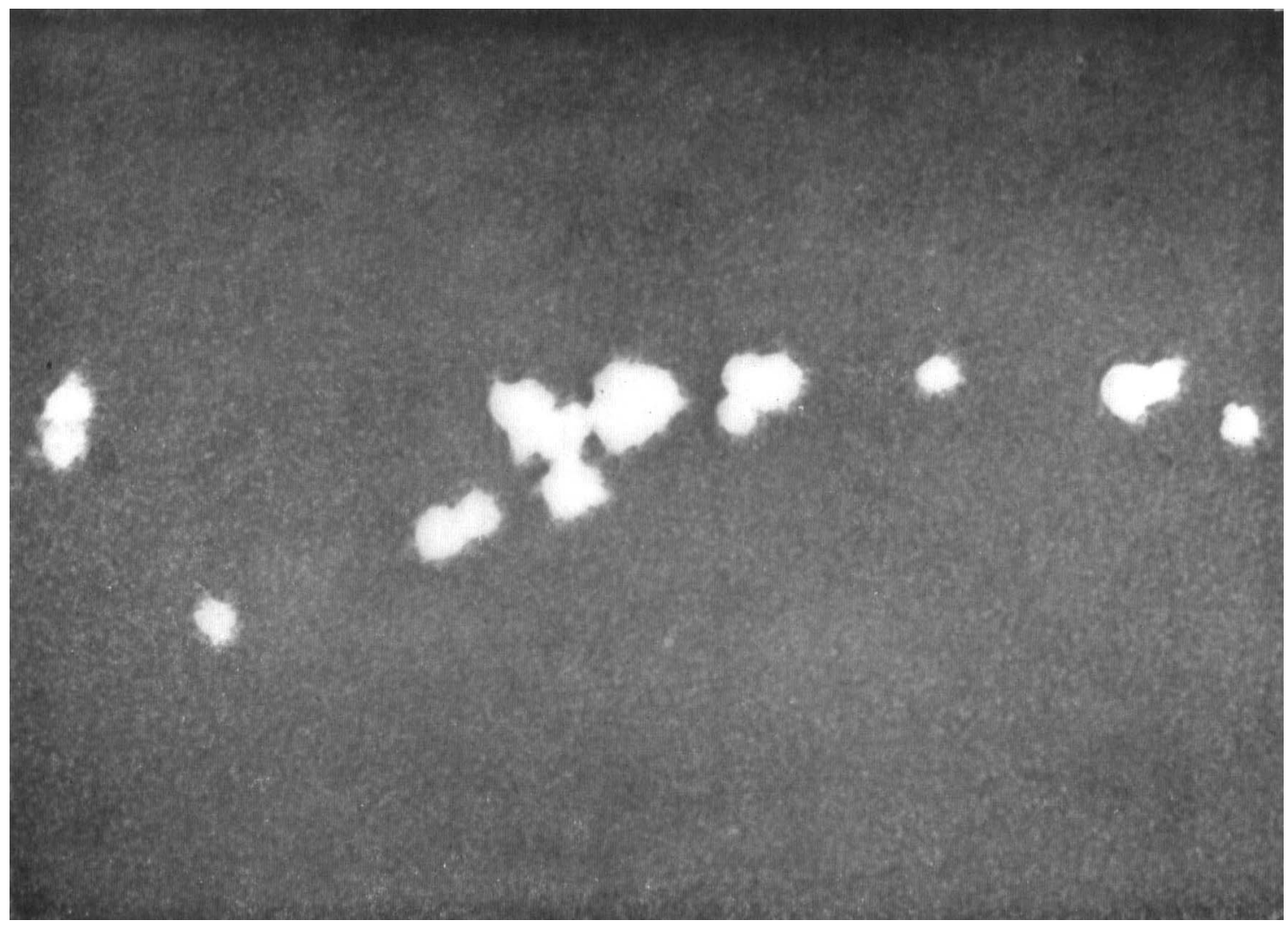

E. S. Anderson and A. Fraskr-'The distribution of adapted particles of Vi-phage II. Plate 1 
Luria, S. E. \& Human, M. L. (1952). A non-hereditary, host-induced variation of bacterial viruses. J. Bact. 64, 557.

Miles, A. A. \& Misra, S. S. (1938). The estimation of the bactericidal power of the blood. J. Hyg., Camb. 38, 732.

Weigle, J. J. \& Bertani, G. (1953). Variations des bactériophages conditionnées par les bactéries hôtes. Ann. Inst. Pasteur, 84, 175.

\section{EXPLANATION OF PLATE}

Clone of phage D1 released during single cycle of growth of phage $A$ in type A of Salmonella typhi. Total number of phage particles in sample after burst: ca. $10^{5}$. Indicator strain type D 1 Salmonella typhi. Magnification, $\times 10$.

(Received 29 February 1956) 